\title{
INSULIN RESISTANCE IN CHRONIC HEPATITIS C VIRUS PATIENTS RECEIVING DIRECT ACTING ANTIVIRAL DRUGS
}

\section{By}

ZAINAB AHMED ALI-ELDIN ${ }^{1 *}$, ESLAM SAFWAT ${ }^{1 *}$, YASSER OMAR ABDEL RAHMAN ${ }^{1}$, MOHAMED SAID ABDELAZIZ ${ }^{2 * *}$ AND EMAD NABIL GEORGE ${ }^{3}$

Department of Internal Medicine, Faculty of Medicine, Ain Shams University ${ }^{1}$, Cairo Postal code 11591, and Department of Endemic Medicine, Faculty of Medicine, Cairo University ${ }^{2}$, Cairo11562, and Department of Internal Medicine, Damnhour Hospital, Ministery of Health and Population ${ }^{3}$, Egypt $\left({ }^{*}\right.$ Correspondence: Zainab_Ahmed@ med.asu.edu.eg.;**eslam_safwat@med.asu.edu.eg.;**msabdelaziz76@yahoo.com)

\section{Abstract}

Association between chronic hepatitis $\mathrm{C}$ (CHC) infection and insulin resistance (IR) is still challenging. The study assessed the impact of using direct acting antivirals (DAAs) on IR and the effect of IR on the treatment response. A total of 101 Egyptian patients with CHC who were eligible to Sofosbuvir based treatment were prospectively involved. All the patients were subjected to baseline liver function test, fasting and post prandial blood glucose, fasting insulin, quantitative HCV PCR and abdominal ultrasound. All the patients received sofosbuvir and daclatasvir with or without ribavirin for 12 weeks. HOMA-IR evaluated IR, both at baseline and at the end of treatment.

The results showed that patients' mean age was $48.59 \pm 10.56$ years; with female dominance (58.4\%). An overall sustained response (SVR) was successfully achieved in 96 patients $(95.04 \%)$. Baseline IR (defined as HOMA-IR >2) was identified in 64 patients $(63.4 \%)$ with a median value of 3.07. Patients with positive sustained virologic response (SVR) had lower median values of HOMA-IR than those with negative SVR; both at baseline and the end of treatment $(p=0.004$, $\mathrm{p}=0.04$; respectively). By the end of treatment, ALT, AST, and FBG showed a significant decrease $(p=0.0001)$. Fasting insulin and HOMA-IR showed a slight improvement $(p>0.05)$.

Keywords: Insulin resistance, HOMA-IR, chronic hepatitis C, direct acting antivirals drugs

\section{Introduction}

Hepatitis $\mathrm{C}$ virus (HCV) infection is a major health problem with a global prevalence of $2.35 \%$, and around 160-170 millions were chronically infected (Lavanchy, 2011). A recent estimate showed a prevalence of $2.8 \%$, equating to more than 185 million infections worldwide (Mohd-Hanafiah et al, 2013). Egypt showed the highest prevalence worldwide (Polaris Observatory HCV Collaborators, 2017) where the HCV genotype 4 is the most prevalent (Sievert et al, 2011). Numerous extrahepatic manifestations have been reported in patients with $\mathrm{HCV}$, suggested a systemic disease rather than just a localized liver disease (Desbois and Cacoub, 2017). Chronic hepatitis C (CHC) is accompanied by glucose metabolic derangements, development of insulin resistance (IR), and type 2 diabetes mellitus which is common in Egypt (El-Tawdy et al, 2016) and measured using the homeostatic model assessment (HOMA) index. Interestingly, euglycemic insulin clamp studies showed that IR in CHC has both a hepatic and a peripheral component i.e., in muscles and was related to viral load but not to liver fat content (Milner et al, 2010).

The underlying mechanism of IR in CHC is still unclear. Some studies suggested an inflammatory role (Lecube et al, 2006). Impaired glucose uptake may also play a role where the surface expression of Glucose Transporters (GLUT1 \& 2) were suppressed in HCV infected cells (Kasai et al, 2009). Insulin resistance is strongly associated with worse outcomes, enhanced fibrosis progression that might be an independent predictor of liver-related mortality in HCV population (Younossi et al, 2013).

The introduction of new interferon-free direct-acting antivirals (DAAs) is associated with the high potency, better tolerability, higher resistance barrier, shortened treat- 
ment dura-tion, all oral regimen, fewer drug interactions and reduced pill burden (Schinazi et al, 2014).

The present study aimed to assess the impact of using direct acting antivirals (DAAs) on insulin resistance (IR) and the effect of IR on the treatment response.

\section{Patients and Methods}

For this prospective cohort study, 101 CHC patients, eligible for DAAs treatment, were included in the period between November 2016 and October 2017 at Ain Shams University Hospitals and Damanhour Hospital. The study was conducted in accordance with the ethical principles of the 1975 declaration of Helsinki, and was approved by the local ethical committee. A written informed consent was obtained from all the participants after explaining the aim and concerns of the study. Eligible patients were 18 years or older and had HCV/PCR positivity. The following patients were excluded: the HBV co-infection, alcoholics, diabetics, any contraindication to DAAs (e.g. hepatocellular carcinoma, extrahepatic malignancy, current or planned pregnancy or lactation, active substance abuse), previous exposure to IFN or DAAs therapy.

All patients were subjected to a thorough clinical examination with estimation of body mass index (BMI), calculated as BMI= Weight $(\mathrm{Kg}) /$ Height squared $\left(\mathrm{m}^{2}\right)$. Baseline laboratory investigations were obtained for proper selection of patients who are eligible for treatment, including complete blood count (CBC), liver and kidney profile, HBsAg, HBcIgG, Alfa-fetoprotein, fasting and post prandial blood glucose, glycated haemoglobin (HbA1c), fasting insulin, quantitative HCV/RNA by the Real-Time PCR using the Cobas Ampli Prep/Cobas TaqMan HCV-RNA assay (Roche Diagnostics; Pleasanton, CA, USA) with the threshold of detection $=15 \mathrm{IU} / \mathrm{ml}$, and the abdominal ultra-sound. IR was determined by HOMA-IR using the following formula: fasting glucose (mmol/L) mutiplied by fasting insulin (mIU/L) divided by 22.5 ; where IR was defined as HOMA-IR >2 (Patel et al, 2011).

According to the national guidelines of $\mathrm{HCV}$ treatment and the nationally available drugs at the study time, patients received either Sofosbuvir/Daclatasvir or Sofosbuvir/ Daclatasvir/Ribavirin (If was difficult to treat; with any of the following: serum albumin $<3.5 \mathrm{~g} / \mathrm{dl}$ and/or total bilirubin $\geq 1.2$ and/or INR $\geq 1.2$ and/or platelet count < $150,000 / \mathrm{mm}^{3}$ ) for 12 weeks.

During treatment period, patients had a regular follw up visits every 2 weeks at the outpatient clinic. $\mathrm{CBC}$, liver and renal function tests were repeated every 4 weeks. A quantitative HCV/PCR was done at the end of treatment, and 12 weeks later to assess SVR. HOMA-IR calculation was repeated at the end of treatment.

Statistical methods: Data were revised, coded, tabulated and introduced to a PC using statistical package for social science (SPSS for Windows version 17 , SPSS Inc., Chicago, IL, USA). Significance level was set at 0.05 . Mean \pm standard deviation (SD) was used for parametric numerical data while median and interquartile range (IQR) for non-parametric numerical data. Frequency and percentage were used to express nonnumerical data.

Chi squared $\left(\chi^{2}\right)$ and Fisher's exact test $(F)$ were used to compare the non-numerical variables, when appropriate. The numerical variables were compared using Student's ttest, Mann Whitney (U) test. Paired t- test and Wilcoxon signed rank test were used to compare numerical values measured twice for the same study group. Spearman correlation test was used to assess the strength of correlation between 2 numerical variables.

\section{Results}

The study included 101 patients with chronic hepatitis $\mathrm{C}$ who were eligible for the DAAs therapy. Their mean age was 48.59 \pm 10.56 years; the females dominant $(58.4 \%)$. BMI ranged between $22.3 \& 30.9$ with a mean of $25.4 \pm 2.27 \mathrm{~kg} / \mathrm{m}^{2}$. Ninety one patients $(90.1 \%)$ received Sofosbuvir/Daclatasvir 
but only ten patients (9.9\%) received Sofosbuvir/Daclatasvir/Ribavirin. An overall SVR was successfully achieved in 96 patients (95.04\%). Baseline IR (defined as HOMAIR > 2) was reported in 64 patients $(63.4 \%)$ with a median value of HOMA-IR 3.07.

The relationship between the baseline IR and patients' personal data was given. IR was significantly higher in females, non smokers and hypertensive patients.

Baseline PCR showed a significant higher median value among males rather than females $(825,500$ vs. 319,$000 ; p=0.003)$. But, there were no significant differences between those with IR and those without regarding PCR. Baseline PCR was positively correlated with ALT, AST; \& negatively correlated with patients' age, FBG, fasting insulin, and HOMA-IR, yet none of the relations was statistically significant ( $p>0.05$ ).
On studying the relationship of HOMA-IR and the acheivement of SVR, the patients with positive SVR had lower median values of HOMA-IR than those with negative SVR; both at baseline and the end of treatment [2.7 (1.3-4.7) vs. 8.8 (5.8-11.3) ; p=0.004 and $2.3(1.5-4.7)$ vs. 18.4 (4.5-21.2); $\mathrm{p}=0.04$; respectively].

ALT, AST, and FBG showed a significant decline at the end of treatment $(52.99 \pm 29.16$ vs. $25.91 \pm 12.29,49.9 \pm 27.5$ vs. $26.13 \pm 9.76$ $\& 93.54 \pm 9.62$ vs. $89.05 \pm 9.12$; respectively) $(\mathrm{p}=0.0001)$. But, fasting insulin and HOMAIR showed a slight improvement [median (IQR):13.4 (6.3-21.2) vs.11.5 (7.2-22.3) \& 3.1 (1.4-5.1) vs. 2.5 (1.6-4.8); respectively], yet, the difference didn't reach statistical significance $(p>0.05)$.

The details were given in tables $(1,2,3,4$ \&5).

Table 1: Relationship between baseline insulin resistance and patients' personal data

\begin{tabular}{|c|c|c|c|}
\hline \multirow[t]{2}{*}{ Personal Data } & \multicolumn{2}{|c|}{ Baseline Insulin resistance } & \multirow[t]{2}{*}{$\mathrm{p}$} \\
\hline & Positive $(n=64)$ & Negative $(n=37)$ & \\
\hline Age (years) & $50.1 \pm 9.7$ & $45.9 \pm 11.6$ & $0.05^{*}$ \\
\hline $\mathrm{BMI}\left(\mathrm{Kg} / \mathrm{m}^{2}\right)$ & $29.9 \pm 1.8$ & $26.2 \pm 2.75$ & $0.013 *$ \\
\hline Male $(\%) /$ Female $(\%)$ & $20(47.6) / 44(74.6)$ & $22(52.4) / 15(25.4)$ & $0.006 * *$ \\
\hline Smoker (\%)/Non-smoker (\%) & $7(36.8) / 57(69.5)$ & $12(63.2) / 25(30.5)$ & $0.008 * *$ \\
\hline Hypertensive (\%)/Non-hypertensive (\%) & $8(100) / 55(59.8)$ & $0(0) / 37(40.2)$ & $0.025^{* * *}$ \\
\hline
\end{tabular}

Table 2: Relation between baseline PCR and personal, ultrasound data and baseline IR

\begin{tabular}{|c|c|c|c|}
\hline \multirow{2}{*}{ Variable } & \multicolumn{2}{|c|}{ Baseline PCR } & \multirow{2}{*}{$\mathrm{p}$} \\
\cline { 2 - 3 } & Median & IQR & \multirow{2}{*}{0.003} \\
\hline Male & 825,500 & $265,000-2,800,000$ & \\
Female & 319,000 & $153,000-910,000$ & \\
\hline Smoker & 495,000 & $180,000-1,070,000$ & \\
Non-smoker & 429,500 & $202,000-1,470,000$ & 0.636 \\
\hline Normal liver by U/S & 484,500 & $215,000-1,320,000$ & \\
Abnormal liver by U/S & 450,000 & $188,110-1,470,000$ & 0.882 \\
\hline Positive IR & 466,000 & $201,500-1,335,000$ & \\
Negative IR & 460,000 & $160,000-1,160,000$ & 0.693 \\
\hline
\end{tabular}

PCR: polymerase chain reaction, IQR: interquartile range, IR: insulin resistance

Table 3: Correlation between baseline PCR and other laboratory parameters

\begin{tabular}{|c|c|c|}
\hline \multirow{2}{*}{ Laboratory parameter } & \multicolumn{2}{|c|}{ Baseline PCR } \\
\cline { 2 - 3 } Age & $\mathrm{r}_{\mathrm{s}}$ & $\mathrm{p}$ \\
\hline ALT & -0.097 & 0.33 \\
\hline AST & 0.083 & 0.407 \\
\hline FBG & 0.095 & 0.346 \\
\hline F. insulin & -0.06 & 0.554 \\
\hline HOMA-IR & -0.06 & 0.551 \\
\hline
\end{tabular}

PCR: Polymerase chain reaction, $r_{s}$ : spearman correlation, FBG: fasting blood glucose, F. insulin: fasting insulin, ALT: Alanine transaminase, AST: Aspartate transaminase, HOMA-IR: Homeostasis model assessment-insulin resistance 
Table 4: Relation between HOMA-IR (at baseline \& end of treatment) and SVR achievement

\begin{tabular}{|c|c|c|c|c|c|}
\hline \multirow{2}{*}{ HOMA-IR } & \multicolumn{4}{|c|}{ SVR } & \multirow{2}{*}{$\mathrm{p}$} \\
\cline { 2 - 5 } & \multicolumn{2}{|c|}{$\mathrm{SVR}+\mathrm{ve}(\mathrm{n}=96)$} & \multicolumn{2}{c|}{$\mathrm{SVR}-\mathrm{ve}(\mathrm{n}=5)$} & \multirow{2}{*}{$\mathrm{IQR}$} \\
\cline { 2 - 5 } & Median & IQR & Median & IQR & \\
\hline At baseline & 2.7 & $1.3-4.7$ & 8.8 & $5.8-11.3$ & 0.004 \\
\hline At end of treatment & 2.3 & $1.5-4.7$ & 18.4 & $4.5-21.2$ & 0.04 \\
\hline
\end{tabular}

SVR: sustained virologic response, IQR: Interquartile range, HOMA-IR: homeostasis model assessment-insulin resistance

Table 5: Changes in main laboratory parameters before and after treatment

\begin{tabular}{|c|c|c|c|}
\hline Laboratory parameter & At baseline & At end of treatment & $\mathrm{p}$ \\
\hline ALT $(\mathrm{M} \pm \mathrm{SD})$ & $52.99 \pm 29.16$ & $25.91 \pm 12.29$ & $0.0001^{*}$ \\
\hline AST $(\mathrm{M} \pm \mathrm{SD})$ & $49.9 \pm 27.5$ & $26.13 \pm 9.76$ & $0.001^{*}$ \\
\hline FBG $(\mathrm{M} \pm \mathrm{SD})$ & $93.54 \pm 9.62$ & $89.05 \pm 9.12$ & $0.001^{*}$ \\
\hline F. insulin [median $(\mathrm{IQR})]$ & $13.4(6.3-21.2)$ & $11.5(7.2-22.3)$ & $0.435^{* *}$ \\
\hline HOMA-IR [median (IQR)] & $3.1(1.4-5.1)$ & $2.5(1.6-4.8)$ & $0.993^{* *}$ \\
\hline
\end{tabular}

*Paired t test, **Wilcoxon signed rankALT: alanine transaminase, AST, aspartate transaminase, FBG: fasting

blood glucose, F. insulin: Fasting insulin, HOMA-IR: homeostasis model assessment-insulin resistance

\section{Discussion}

Many studies evaluated the association between HCV chronic infection and IR, yet, the results were conflicting. With the development of the DAA therapies, HCV can be successfully eradicated; with high safety and efficacy (Dhingra et al, 2014). The mutual effect between DAA HCV treatments and IR still has to be cleared..

In the present study, the prevalence of IR among cases was $63.4 \%$, a result that was contradictory to a recent Iranian study which recorded a much lower prevalence $(26.9 \%)$ and declared that the CHC could not be considered a risk factor for IR (Eshraghian et al, 2017). This difference might be explained by the difference in genotypes as genotype 4 is most prevalent among Egyptian patients while genotype 1 and 3 are the most frequent in Iran.

The relationship between IR \& HCV genotype is still debatable. Hui et al. (2003) reported that patients with genotype 3 had significantly lower HOMA-IR than other genotypes even after the adjustement of other variables. Despite similarity in the genotype 4, in a cohort of Egyptian patients with CHC, IR was reported in only $31 \%$ of nondiabetic patients (Amer et al, 2016). It seemed that the difference might be due to their use of a higher cut off of HOMA-IR > 3.0 .

In agreement with the present study, when using a cut-off level similar to (HOMA $\geq 2$ as indicating IR), Péres et al. (2013) showed the prevalence of IR was similarly high (65\% \& $57 \%$ in genotype $1 \&$ genotype 3 , respectively) So, it worthnoting that using diffierent HOMA-IR cut-off levels to define IR played a major role in heterogeneity in the prevalence results.

Generally, it is well known that males poses a higher risk for IR and diabetes, but the protective effect of female decreases with age, particularly in women above the age of 50, considered an average age for menopause (Gayoso-Diz et al, 2011). In the present study, female patients were the predominant in having IR $(\mathrm{p}=0.006)$; a finding that might be attributed to the older age of the present patients (mean age $48.59 \pm 10.56$ years).

Consequently, the females were associated with directly measured IR in the setting of HCV infection (Mukhtar et al, 2013). Of note, HCV itself increases the risk of IR, thus, the sex effect on IR parameters might be overwhelmed by the complex interplay between virus and host in a way that differs from the non-HCV population.

The present study showed a significant impact of HOMA-IR on acheiving the SVR, where the median values of HOMA-IR (both at baseline and at the end of treatment) were significantly lower in the patients who had positive SVR ( $p=0004,0.04$; respectively). To the present author's knowledge, no recent data is available about the effect of IR on SVR in patients receiving new DAAs.

The present results agreed with Saady et 
al. (2013) in Egypt who showed that pretreatment IR was an independant negative predictor of SVR (OR 1.15; $\mathrm{p}<0.001$ )

Moreover, two published meta-analyses examined the effect of IR on SVR including fourteen studies with more than 2700 patients. Some of these studies did not find an association between IR and achievement of SVR; with a baseline HOMA-IR less than 3 and a low prevalence of advanced fibrosis (Eslam et al, 2011; Deltenre et al, 2011). Besides, IR did not appear to be strongly associated with SVR $(p=0.68)$, even after controlling for genotype; the odds ratio for the association between IR and SVR was not altered as $1.02(0.89-1.16)$; $\mathrm{p}=0.81$ (Brandman et al, 2012). Notably, the standard of care at the time of all these studies was pegylated IFN and ribavirin and not DAA.

At the end of treatment, there was a reduction in mean value of FBG, median values of fasting insulin and HOMA-IR [93.54- \pm 9.62 vs. $89.05 \pm 9.12,13.4(6.3-21.2)$ vs. 11.5 (7.2-22.3), $3.1(1.4-5.1)$ vs. $2.5(1.6-4.8) ; \mathrm{p}=$ $0.0001,0.435,0.993$; respectively].

These results went with the study which elucidated the mutual impact between the treatment response and parameters of the glucose abnormalities after DAAs therapy in $\mathrm{CHC}$ patients. It was found that the mean IR declined at the follow up end from $2.7 \pm 2.9$ to $2.6 \pm 1.8(\mathrm{p}=0.75)$ but without significantly different (Huang et al, 2017).

In a retrospective study evaluating 149 $\mathrm{HCV}$-positive diabetic patients who received different DAA regimens, FBG analysis revealed a significant reduction during treatment $(\mathrm{p}=0.007)$; with a reduction mean value $-52.86 \mathrm{mg} / \mathrm{dL}$. The results were neither dependent on the DAA regimen nor HCV genotype (Pavone et al, 2016).

\section{Conclusion}

The insulin resistance (IR) was highly prevalent among Egyptian patients with Chronic Hepatitis $\mathrm{C}$ with a negative impact on SVR.

The use of DAAs may ameliorate IR at the end of treatment using DAAs.
CHC among Egyptian patients is accompanied by a high prevalence of IR. Furthermore, IR has a negative impact on SVR; yet, just using DAAs in treating HCV may ameliorate IR at the end of treatment even before reaching SVR. Such final conclusions have to be confirmed by controlled clinical trials with long-term follow-up.

\section{Compliance with ethical standards}

All authors declared that they neither received fund nor have any conflict of interest.

All procedures performed were in accordance with the ethical standards of the institutional and national research committee and with the 1975 Helsinki declaration and its later amendments. Informed consent was obtained from all individual participants included in the study

\section{References}

Amer, AF, Baddour, MM, Elshazly, MA, Fadally, G, Hanafi, NF, et al, 2016: Study of prevalence and effects of insulin resistance in patients with chronic hepatitis C genotype 4 . East. Mediterr. Hlth. J. 21:803-10.

Brandman, D, Bacchetti, P, Ayala, CE, Maher, JJ, Khalili, M, 2012: Impact of insulin resistance on $\mathrm{HCV}$ treatment response and impact of $\mathrm{HCV}$ treatment on insulin sensitivity using direct measurements of insulin. Diabetes Care 35: 1090-4.

Deltenre, P, Louvet, A, Lemoine, M, Mourad, A, Fartoux, L, et al, 2011: Impact of insulin resistance on sustained response in HCV patients treated with pegylated interferon and ribavirin: a meta-analysis. J. Hepatol. 55:1187-94.

Desbois, AC, Cacoub, P, 2017: Diabetes mellitus, insulin resistance and hepatitis $C$ virus infection: A contemporary review. World J. Gastroenterol. 23:1697-711.

Dhingra, A, Kapoor, S, Alqahtani, SA, 2014: Recent advances in the treatment of hepatitis $\mathrm{C}$. Discov. Med. 18:203-8.

El-Tawdy, AHF, Ibrahim, EA, Abdallah, ES, Al Sakhawy EMA, Morsy, TA, 2016: Screening for the diabetes mellitus: General information for patients to avoid foot amputation. EMMJ, 71, 1:61-74

Eshraghian, K, Lankarani, KB, Fattahi, MR, Esmailnejad, A, Peymani, P, 2017: Low prevalence of insulin resistance among Iranian Patients with Chronic Hepatitis C Virus Infection: A 
Case-Control Study. Curr. Diabetes Rev. [Epub ahead of print].

Eslam, M, Aparcero, R, Kawaguchi, T, Del Campo, JA, Sata, M, et al, 2011: Metaanalysis: Insulin resistance and sustained virological response in hepatitis C. Aliment. Pharmacol. Ther. 34:297-305.

Gayoso-Diz, P, Otero-Gonzalez, A, Rodriguez-Alvarez, MX, Gude, F, Cadarso-Suarez, C, et al, 2011: Insulin resistance index (HOMA-IR) levels in a general adult population: curves percentile by gender and age: The EPIRCE study. Diabetes Res. Clin. Pract. 94:146-55.

Huang, JF, Huang, CF, Yeh ML, Dai, CY, Hsieh, MH, Yang, JF, et al, 2017: The outcomes of glucose abnormalities in chronic hepatitis $\mathrm{C}$ patients receiving interferon-free direct antiviral agents. Kaohsiung J. Med. Sci. 33:56771.

Hui, JM, Sud, A, Farrell, GC, Bandara, P, Byth, K, et al, 2003: Insulin resistance is associated with chronic hepatitis $\mathrm{C}$ virus infection and fibrosis progression [corrected]. Gastroenterology 125:1695-704.

Kasai, D, Adachi, T, Deng, L, Nagano-Fujii, M, Sada, K, et al, 2009: HCV replication suppresses cellular glucose uptake through downregulation of cell surface expression of glucose transporters. Hepatology 50:883-94.

Lavanchy, D, 2011: Evolving epidemiology of hepatitis C virus. Clin. Microbiol. Infect. 17: 107-15.

Lecube, A, Hernández, C, Genescà, J, Simó, R, 2006: Proinflammatory cytokines, insulin resistance, and insulin secretion in chronic hepatitis C patients: A case-control study. Diabetes Care 29:1096-101.

Milner, KL, van der Poorten, D, Trenell, M, Jenkins, AB, Xu, A, et al, 2010: Chronic hepatitis $\mathrm{C}$ is associated with peripheral rather than hepatic insulin resistance. Gastroenterology 138: 932-41.

Mohd-Hanafiah, K, Groeger, J, Flaxman, AD, Wiersma, ST, 2013: Global epidemiology of hepatitis $\mathrm{C}$ virus infection: new estimates of age-specific antibody to HCV seroprevalence. Hepatology 57:1333-42.
Mukhtar, NA, Bacchetti, P, Ayala, CE, Melgar, J, Christensen, S, et al, 2013: Insulin sensitivity and variability in hepatitis $\mathrm{C}$ virus infection using direct measurement. Dig. Dis. Sci. 58:1141-8.

Patel, K, Thompson, AJ, Chuang, WL, Lee, CM, Peng, CY et al, 2011: Insulin resistance is independently associated with significant hepatic fibrosis in Asian chronic hepatitis C genotype 2 or 3 patients. J. Gastroenterol. Hepatol. 26:11828.

Pavone, P, Tieghi, T, d'Ettorre, G, Lichtner, M, Marocco, R, et al, 2016: Rapid decline of fasting glucose in HCV diabetic patients treated with direct-acting antiviral agents. Clin. Microbiol. Infect. 22:462-8.

Péres, DP, Cheinquer, H, Wolf, FH, Cheinquer, N, Falavigna, M, et al, 2013: Prevalence of insulin resistance in chronic hepatitis $\mathrm{C}$ genotype 1 and 3 patients. Ann. Hepatol. 12:871-5.

Polaris Observatory HCV Collaborators, 2017: Global prevalence and genotype distribution of hepatitis $C$ virus infection in 2015: A modelling study. Lancet Gastroenterol. Hepatol. 2:161-76.

Saady, Y, Ahmed, A, Saleh, DA, Doss, W, 2013: Adipokines and insulin resistance, predictors of response to therapy in Egyptian patients with chronic hepatitis $\mathrm{C}$ virus genotype 4 . Eur $\mathrm{J}$. Gastroenterol. Hepatol. 25:920-5.

Schinazi, R, Halfon, P, Marcellin, P, Asselah, T, 2014: HCV direct-acting antiviral agents: the best interferon-free combinations. Liver Int. J. 34, 1:69-78.

Sievert, W, Altraif, I, Razavi, HA, Abdo, A, Ahmed, EA, et al, 2011: Systematic review of hepatitis $\mathrm{C}$ virus epidemiology in Asia, Australia and Egypt. Liver Int. J. 31, 2:61-80.

Younossi, Z, Negro, F, Serfaty, L, Pol, S, Diago, M, et al, 2013: Homeostasis model assessment of insulin resistance does not seem to predict response to telaprevir in chronic hepatitis $\mathrm{C}$ in the REALIZE trial. Hepatology 58:1897906. 